\title{
Genetic and Epigenetic Instability of Human Pluripotent Stem Cells
}

\author{
Tung-Liang Chung, Nilay Y. Thakar and Ernst J. Wolvetang* \\ Stem Cell Engineering Group, Australian Institute for Bioengineering and Nanotechnology, Level 4, Building 75, \\ University of Queensland, St Lucia QLD 4072 Australia
}

\begin{abstract}
The preservation of the genetic and epigenetic integrity of human embryonic stem cells (hESCs) and induced pluripotent stem cells (iPSCs) during in vitro propagation is critical for their use in both research and future therapeutic applications. It has been reported that hESCs and iPSCs have the ability to adapt to various culture conditions. However, human pluripotent stem cells cultured in serum free media can frequently accumulate point mutations, aneuploidy, and progressive epigenetic changes over prolonged culture in vitro for reasons that are poorly understood. The phenotypic and epigenetic changes brought about by the culture conditions can have significant impacts on their use in research and in clinical applications. An increased understanding of the potential effects of in vitro culture environments on pluripotent stem cell growth can enhance the development of improved culture systems for hESCs or iPSCs, and facilitate any future therapeutic applications using these cells. In this review, we first focus on the occurrence, potential causes and consequences of genetic and epigenetic unstable human pluripotent stem cells in vitro. We further discuss the current methods for detection and characterization of abnormal pluripotent stem cells that involve simply traditional karyotype analysis. All these observations highlight the need for novel screening strategies to determine the safety of hESCs or iPSCs and optimization and standardization of procedures for the generation and culture of pluripotent stem cells that minimize culture-induced epigenetic and genetic instability.
\end{abstract}

Keywords: Pluripotent stem cells, epigenetic, karyotype, induced pluripotent stem cells, aneuploid.

\section{INTRODUCTION}

Since the publication of the first report on the derivation and characterization of human embryonic stem cells (hESCs) in 1998 [1], there has been considerable progress in the development of methodologies for their growth, genetic manipulation, and differentiation into a variety of cell types. However, significant challenges remain before hESCs can become a robust cellular platform for regenerative medicine.

As hESCs can potentially differentiate into all the cell types that make up a human body, they possess exciting potential as a source of cells for regenerative medicine, and are valuable tools for drug discovery and understanding human development and disease. However, translating these advantages into clinical benefits faces many challenges including (1) efficient and cost effective differentiation into the desired cell types, (2) ensuring the absence of potentially tumorigenic undifferentiated hESCs from the final product, (3) absence of potential pathogenic animal derived factors, and finally, (4) maintaining genetic stability during longterm culture. It is this final safety issue that will form the focus of this review.

One of the key issues impeding the application of hESCs in regenerative medicine is the occurrence of karyotypic instability of hESCs during long term culture [2, 3]. Indeed, induced pluripotent Stem Cells (iPSCs) which have been widely acclaimed to overcome the immune rejection and

*Address correspondence to this author at the Stem Cell Engineering Group, Australian Institute for Bioengineering and Nanotechnology, Level 4, Building 75, University of Queensland, St Lucia QLD 4072 Australia; Tel: 61-7-33463894; E-mail: e.wolvetang@uq.edu.au ethical issues faced by hESCs [4] appear to exhibit a similar unstable genotype. Although the occurrence and potential consequences of genetic instability for cell therapy has been recognized to some extent, very little is known about its causes. It has been widely proposed that the process of adaptation of pluripotent stem cells to in vitro culture conditions critically contributes to development of karyotypic abnormalities [5]. Within the context of this proposed adaptation model, it remains unclear what parameters, or combination of parameters, imposed by the in vitro culture method might affect genetic stability of hESCs. For example, conclusive evidence as to whether individual factors such as oxygen tension, medium components, extracellular matrix ECM or mechanical versus enzymatic propagation affect genetic stability of hESCs in culture remains elusive, let alone how such factors would bring about genetic instability. A detailed understanding of the effects of in vitro culture environments on hESC growth is required for the development of improved culture systems for hESCs or iPSCs, and a prerequisite for any future therapeutic applications using these cells.

\section{GENETIC INSTABILITY OF HUMAN PLURIPO- TENT STEM CELLS}

\section{Occurrence of Karyotypically Abnormal Human Pluripotent Stem Cells in vitro}

Long-term maintenance of stem cells in an environment potentially suboptimal compared with the in vivo situation may lead to the accumulation of genetic defects. It should be considered that forcing embryonic stem cells to expand in vitro, even if this is carried out under empirically determined 
"optimal" culture conditions, may lead to adaptive genetic change by itself, given that, in vivo, the inner cell mass exists in a pluritotent state for only a few days before forming the tissues of the fetus [6]. Indeed, it is likely that most of the hESC and iPSC lines in use today carry genetic and epigenetic artifacts of accommodation to tissue culture [4] and that the type and number of abnormalities varies from laboratory to laboratory due to differences in culture methods. Although, as discussed later in this review, point mutations, small deletions and amplifications and epigenetic changes are not routinely monitored in hESC and iPSC lines, gross genetic changes, such as variations in chromosomal number and chromosomal imbalance in hESCs and iPSCs can be observed by simple G-banding karyotype analysis and alter the behavior and gene expression patterns of hESC lines. Such gross karyotypic abnormalities are of particular relevance if stem cell derivatives are going to be used in cell therapies, as the relationship between genetic instability and carcinogenesis is well established [7-9].

Various chromosomal abnormalities have been observed by numerous groups in multiple hESC lines over extended time in culture, with prevalence for aneuploidy of chromosome 12, 17, and X. These karyotypic abnormalities are also frequently observed in human embryonal carcinoma cells, the malignant counterpart of embryonic stem cells $[2,3,10-$ 14]. A propensity for the clonal selection of trisomy 12 was reported in HUES [11] and $\mathrm{H} 1$ [14] lines, whereas trisomy 17 was also observed $[10,14]$ in BG01 and BG02. Preferential gains in 12 or 17 in $\mathrm{H} 14$ have however varied between laboratories [15] as Rosler et al. [16] reported trisomy 20 as the prevalent aneuploidy in $\mathrm{H} 1, \mathrm{H} 7$ and $\mathrm{H} 9$. Trisomy of chromosomes 13 and 3 was only observed in SA002 [17, 18] and in Miz-hES13 [19], respectively. The evidence therefore suggests that there is a preponderance of chromosome 12 and 17, but other abnormalities can also occur, depending on the hESCs analyzed. It is not unlikely that the karyotypic abnormalities in fact occur randomly, but that only those genetic abnormalities that provide advantages of growth and survival to hESCs are selected for over time in culture. As such the emergence of a particular type of chromosomal abnormality may depend on the way the hESCs were cultured.

While genetic abnormalities have been thoroughly investigated in hESCs, the occurrence of karyotypic abnormalities in human iPSCs has been largely ignored thus far [20]. Only recently Aasen et al. [21] showed that continuous passaging of human iPSCs resulted in frequent chromosomal abnormalities [karyotype 46,XY,t(17;20)(p13;p11.2)] starting as early as weekly passage 13 . This observation is of concern since karyotypic abnormalities are usually not observed at such early passages in hESCs. This finding therefore suggests that the long-term culture of human iPSCs, much like hESCs, has to be monitored carefully for any genetic abnormality.

Of concern in iPSC generation is the viral-gene delivery which can potentially cause genetic abnormalities; however, these can be easily overcome by the recent advancements such as non-viral gene-delivery methods [22-24] and piggyBac transposon methods [25]. Culture methods such as inclusion of Valproic acid [26] and inhibition of critical cell signaling pathways such as p53 [27-31], MAPK [32] and GSK3 have been widely used recently to enhance reprogramming efficiency. However, Marion and colleagues show that p53 is critically involved in preventing the reprogramming of cells carrying various types of DNA damage, including short telomeres, DNA repair deficiencies, or exogenously inflicted DNA damage. Reprogramming in the presence of pre-existing, but tolerated, DNA damage is aborted by DNA damage response activation and p53dependent apoptosis. Therefore, inhibition of p53 allows for efficient reprogramming in the face of potential DNA damage and chromosomal abnormalities. These observations indicate that during reprogramming, cells can increase their intolerance to different types of DNA damage and that p53 is critical in preventing the generation of human and mouse pluripotent cells from suboptimal parental cells [33]. Most notably, current reprogramming protocols require cells to be kept in culture for extended periods of time (up to 20 passages) post reprogramming, presumably to allow further stabilization of the epigenome of correctly reprogrammed clones. It is most likely that under such selective pressure the risk of selection for genetically abnormal growth advantaged clones is increased. Therefore, in particular in the case of iPSCs, culture conditions may play an increasingly significant role in contributing to the emergence of genetic abnormalities.

It is widely recognized that for regenerative therapy using iPSCs to be effective and clinically relevant, much faster and efficient ways of reprogramming will need to be developed. However, if the price paid for this is a loss of genetic integrity which ultimately leads to the generation of genetically abnormal patient-specific cell lines, that can in no way be used for regenerative medicine. Therefore such methodologies may need to be re-examined and a clear set of detailed protocols must be put in place to ensure consistent safety of iPSCs for their application in regenerative medicine.

\section{Aneuploid hES Cells and EC Cells}

Aneuploid hESCs, or cultured-adapted hESCs as reviewed by Baker et al. [34], grow faster and are highly clonogenic as compared to their diploid counterpart $[11,35$ 37]. This may be related to the fact that aneuploid hESCs express higher level of the hESC markers, SSEA-3 and SSEA-4, [35, 38], and pluripotency genes, OCT4 and NANOG. They also up-regulate self-renewal signaling pathways involving Nodal/Activin, Wnt, FGF, Hedgehog and down-regulate differentiation pathways involving BMP and Notch [14, 35, 38]. Despite these imbalances of signaling pathways associated with self-renewal, aneuploid hESCs seem to maintain the ability to differentiate in vitro $[2,10]$ and in vivo [37] but with different efficiency. Indeed, the presence and isolation of undifferentiated cells from the teratoma generated by the injection of the aneuploid BG01V and HUES-3 cell lines may indicate the formation of an undifferentiated teratocarcinoma-like tumour and not the completely differentiated benign teratoma [36, 37]. In particular, Plaia et al. identified a few small pockets of OCT$3 / 4+$ cells by immunochemistry, indicating the persistence of undifferentiated cells 8-12 weeks after injection of BG01V. Further study showed that these hES-like cells that were OCT-3/4+, SSEA-4+, and TRA-1-60+ could be isolated and expanded in culture from a 12 -week old teratocarcinoma-like 
tumour [36]. Teratocarcinomas, a subset of germ cell tumors (GCT), are malignant tumors comprising both somatic tissues and undifferentiated malignant stem cells, identifiable as embryonal carcinoma (EC) cells.

Aneuploid hESCs and EC cells indeed share many features. For example, both are pluripotent and express pluripotency-related genes such as NANOG, OCT4, SOX2, TRA-1-60. In addition, they display a reduced tendency to undergo apoptosis, increased proliferation, and increased clonal growth [39]. They also show reduced capacity for differentiation by forming teratocarcinoma when injected in immune-deficient mice, suggesting an increased propensity for self-renewal [5, 40-42]. Furthermore, they acquire progressive genetic changes that show striking similarity (i.e. gains of chromosome 12, 17 and X) [34]. Thus, progressive adaptation of hESCs in culture can result in cells with hallmarks of EC cells from teratocarcinoma, while still retaining the capacity for differentiation. These observations led Baker et al. to suggest that aneuploid hESCs may appear in culture by ways that mimic changes occurring in EC cells during tumor progression [34]. Since the typical karyotypic changes in aneuploid hESCs mirror those found in their malignant counterparts from teratocarcinomas, the mechanisms that drive adaptation and selection of such variants in vitro may give insights into the mechanisms underlying GCT progression in vivo. Indeed, since human embryonic germ cell lines (hEGCs) have been successfully derived [43-45], it would be of great interest to determine whether these cultured hEGCs display similar adaptation process as that observed in aneuploid hESCs and EC cells, as EC cells are derived from germ cell tumors (GCT), which is the malignant counterpart of PGCs. Even though the obvious parallels are between aneuploid hESCs and EC cells, since the growth conditions of a cancer cell in vivo and a stem cell in vitro are not the same, it is possible that the causes of karyotypic abnormalities in hESCs in vitro might not be applicable to the occurrence of genetic instability of EC cells in vivo.

Based on the similarity between aneuploid hESCs and EC cells, Andrews et al. [39] proposed that EC cells from teratocarcinomas and diploid ES cells from embryos might be regarded as existing at different points along a continuous spectrum of adaptation, from complete "normality" at one end (represented by the ICM cells within an embryo) to extreme "abnormality" at the other (represented by a EC cell from a GCT. Although this hypothesis seems to imply that the appearance of karyotypic abnormalities is inevitable under in vitro culture conditions, a scenario that would spell the end of hESC and human iPSC (hiPSC) based therapies, it highlights the need for increased understanding of the biology of hESCs and the molecular mechanism that drive self-renewal and commitment to differentiation as well as the influences of culture environments on hESC growth and thus develop novel improved culture methods that minimize the selective advantage of variant cells.

\section{Potential Consequences of Karyotypic Abnormalities in hESCs}

The most frequent karyotypic changes observed in aneuploid hESCs are gains of chromosome 12, 17, and to a lesser extent $\mathrm{X}$, in which similar nonrandom changes are also seen in EC cells. The high frequency of these abnormalities indicates that these chromosomes may contain genes critical for cell growth and potentially tumorigenesis. For example, repeated nonrandom gain of $17 \mathrm{q}$ in aneuploid $\mathrm{hESCs}$ has also been reported in other cancer types such as breast cancer [46], indicating that this region may contain a number of gene(s) that are important to the malignant cells. One candidate gene located in this region is Survivin. Survivin is an antiapoptotic gene, which is expressed in neuroblastomas and is particularly associated with the highest-rick tumors [47]. In addition, the pluripotency gene NANOG is located on $12 p$ in the 12 p13 band. Overexpression of a gene such as NANOG, which tends to promote self-renewal and prevent differentiation, could provide cells with an advantage in culture. The other stem cell-associated genes, DPPA3 and GDF3, are also located in the 12 p13 band as well as the cellcycle regulator CCND2. Thus such genes are potential players in culture adaptation. The genes for the androgen receptor (AR) and its interacting protein NONO [48] are also located on chromosome $\mathrm{X}$. AR is involved in cell cycle progression [49], with influence over cell division and apoptosis. The individual biological effect of expression of the genes in aneuploid hESCs mentioned above however needs to be further determined. What other genes provide a selective advantage in hESCs that display trisomy of chromosome $1,3,7,8,9,14$, and 20 remains to be determined. Neither is it clear whether a mutation, or other genetic or epigenetic change that provides a selective advantage for the undifferentiated stem cell, would necessarily have any effect on the behavior of specific differentiated derivatives, until it is addressed experimentally.

\section{Detection of Aneuploid hESCs}

The conventional analysis of genome integrity is based on $\mathrm{G}$ banding of metaphase spreads. This technique, although reliable when performed by an appropriately trained operator, does not allow the detection of microdeletions or mutations. A recent extensive study using comparative genome hybridization $(\mathrm{CGH})$ demonstrated that 5 of 10 hESC lines analyzed presented ploidy alterations (including deletions and amplifications) during prolonged time in culture [3]. Of note is the observed amplification of the proto-oncogene, MYC, that is likely to be associated with a selective growth advantage for hESCs, because this transcription factor affects cell proliferation via cell cycle progression [50]. Overexpression of MYC has also been observed in the spontaneous transformation of murine and human adult mesenchymal stem cells in vitro $[3,51]$. Furthermore, mitochondrial DNA mutations, undetectable by G-banding, have also been observed in $2 / 10$ cell lines in the study, and it is of interest that these are a common feature of ageing and cancer [52, 53]. Thomson et al. [54] also recently reported that, one hESC line $\mathrm{H} 9$ did not display gross karyotypic abnormalities, but $\mathrm{CGH}$ suggested the presence of random deletions clustered near the telomeres of many chromosomes. Additionally, Werbowetski-Ogilvie et al. showed that two variant hESC lines (v-hESC-1 and v-hESC2) that express pluripotency markers at high levels and do not harbor chromosomal abnormalities by standard cytogenetic measures, exhibit amplification at 20q11.1-11.2 in vhESC-1 and a deletion at 5q34a-5q34b;5q3 and a mosaic gain of chromosome 12 in v-hESC-2 when analysed by 
comparative genomic hybridization. Indeed, these two lines possess some features of neoplastic progression, including a high proliferative capacity, growth-factor independence, a 9to 20 -fold increase in frequency of tumor-initiating cells, niche independence and aberrant lineage specification [55]. These reports clearly indicate that, in addition to G-banding, the use of robust molecular and cytogenetic tools such as Fluorescence In Situ Hybridization (FISH), Multicolor Spectral Karyotype (SKY-FISH) and Comparative Genomic Hybridization (CGH) are important to facilitate the analysis of potential chromosomal aberrations. Only a combination of different techniques, as well as functional characterization to distinguish partially transformed and normal pluripotent stem cells, can ultimately guarantee good coverage of all possible genetic abnormalities [55-57]. The occurrence and potential detrimental effect of karyotypic alterations must be addressed over a long period of time when designing culture conditions for hESC maintenance. The karyotype analysis at passage around 20-30 showing diploid karyotype may not be sufficient to demonstrate the genetic stability of hESCs over time (i.e. more than 50 or 100 passages) in culture.

\section{Potential Causes and Mechanisms of Development of Karyotypic Abnormalities in Cultured hESCs and iPSCs}

Any cell that is not committed to differentiate is faced with three simple fate choices: differentiation, death or selfrenewal. For example, within the embryo and the developing gonad, PGC are committed to the reproductive lineage, but they retain the potential to give rise to pluripotent cells [44, 58]. Thus, their differentiation pathways must be tightly controlled so as to ensure the correct development of gametes. It is widely accepted that carcinoma in situ (CIS) is the precursor for GCT, which might then be regarded as the first step in the malignant transformation that leads to the loss of differentiation control that normally acts in PGC [59]. GCT are thought to initiate in utero [60]. It has been suggested by Harrison et al. [5] that changes in the microenvironment may play a key role in CIS development from PGC, either by delaying their differentiation and allowing adaptation to the changed conditions, or because the altered conditions fail to provide factors important to PGC fate control. Likewise, in cultured aneuploid hESCs, differentiation capacity might be aberrantly affected by the removal of the fate control signal that may be present in the ICM of the embryo. Indeed, our group has previously reported that withdrawal of b-FGF (a medium additive to maintain growth of undifferentiated hESCs [61]) from hESC media induced differentiation in the normal but not in the abnormal cells, suggesting altered control of differentiation in the aneuploid hESCs [37].

Control of differentiation can be altered even by a small increase in the probability of self-renewal and such imbalance of self-renewal and differentiation can be induced by genetic changes. In somatic cells, DNA damage may be repaired following cell cycle arrest at the G1 checkpoint. However, mouse and primate ESCs lack a G1 checkpoint, and variant cells are proposed to be subjected to an apoptotic fate $[62,63]$. Indeed, aberrations do occur during hESC replication. The most frequent karyotypic changes so far reported involved chromosome trisomy, indicating that chromatid separation during mitosis may be prone to error in hESCs. Damelin et al. [64] recently reported that chromo- some gain and loss during the ex vivo culture of human and mouse ESCs could be a consequence of the inefficient G2 decatenation checkpoint, which delays entry into mitosis from $\mathrm{G} 2$ if the chromosomes have not been sufficiently disentangled or decatenated, suggesting that poor mitotic control may be a general feature of embryonic stem cells. Further, the study by Mantel et al. [65] revealed that the mitotic-spindle assembly checkpoint (SAC), which helps maintain chromosomal integrity during all cell divisions, is functional in mouse ES cells and hESCs, but does not initiate apoptosis as it does in somatic cells. This allows an unusual tolerance for mitotic failure-induced polyploidy, which is common in rapidly proliferating cell populations and which is also common in human neoplastic disease. Checkpoint activation in ESC-derived early differentiated cells, however, results in robust apoptosis without polyploidy/aneuploidy similar to that in somatic cells. They suggested that ESCs display intrinsic absence of checkpoint-apoptosis coupling. Because SAC is crucial during every cell division and because mitotic errors often occur in rapidly proliferating cells, the coupling is important for genome maintenance. Thus, inefficiency of G2 decatenation checkpoint and uncoupling of checkpoint-apoptosis in hESCs might be a likely source of karyotypic abnormalities. This clearly suggests that an altered apoptotic mechanism caused by in vitro culture environments may have great impact on the preservation of genetic integrity of hESCs.

The aneuploidy observed in hESCs is also most likely driven by the stresses induced by variable environmental conditions to which these cells are exposed in culture. As suggested by Baker et al. these aberrations may be driven by stresses induced by culture conditions that exert selective pressures driving the emergence of the variant populations [34]. Continuing studies will be necessary to determine the exact causes of the abnormalities, and how to minimize the appearance of abnormal cells by altering the in vitro culture components (i.e. oxygen tension, medium components, ECM mechanical versus enzymatic propagation).

It has been proposed that passage of hESCs by mechanical dissection preserves genomic integrity better than bulk techniques, involving non-enzymatic (cell dissociation buffer) or enzymatic (collagenase or trypsin) methods [10, 14, 66]. This suggestion seems plausible as hESCs express gap junctions and cell adhesion molecules indicating that cell-tocell contacts are essential for their function [18, 67-69]. Mechanical passage of hESC colonies allows preservation of these connections, given that only a portion of the colony is dissected and re-plated for successive culture with this method. In contrast, bulk techniques cause destruction of cell contacts to varying extent (i.e. trypsin more than collagenase) and therefore may impose a survival pressure on the isolated hESCs in culture. In contrast to the argument that dissociating to small clumps or single cells leads to increased chance of karyotypic abnormalities, it has been observed that sporadic aneuploidies can also occur in mechanically passaged cells cultured with $20 \%$ Knock-out serum replacement (KSR) and $4 \mathrm{ng} / \mathrm{ml}$ b-FGF $[18,66]$.

In vivo the ICM, from which hESCs are derived, experiences low oxygen tension. Exposure of hESCs to ambient oxygen tension $(20 \%)$ could promote abnormalities by causing damage to chromosomes (shortening telomeres) and 
mitochondrial DNA [71]. Maitra et al. [3] found alterations in mitochondrial DNA in late-passage hESCs cultured under $20 \%$ oxygen tension. Furthermore, Forsyth et al. [72] showed that hESCs cultured under room oxygen tension displayed more genetic changes than the cells cultured under physiological oxygen (2\%), as compared to their parental lines. These observations imply that the high oxygen tension could be responsible for promoting chromosome abnormalities, probably due to oxidative stress. However, larger numbers of cell lines examined are required to draw definite conclusions.

It is known that culture conditions influence gene expression and, hence, probably many properties of the cells. For example, Koivisto et al. [73] recently reported that hESCs cultured in knock-out serum replacement (KOSR) containing medium display an increased growth rate compared with those grown in fetal calf serum (FCS) -containing medium. To further understand the mechanisms supporting this enhanced growth of hESCs in KOSR-containing medium, Skottman et al. [74] performed gene expression profiling using DNA microarrays to compare whole genome gene expression changes between hESCs cultured in serumand KOSR-containing medium. Among the genes expressed at similar levels in cells cultured in either medium, there are many ESC markers, such as OCT-3/4, NANOG, Cripto, and DNMT3B, suggesting a non-pluripotency related cause for enhanced growth in serum replacement. Several genes involved in the regulation of transcription, RNA processing, and cell proliferation were upregulated in the cells cultured in KOSR medium. For example, the downregulation of SULF1 expression may enhance growth signaling in cancer, and cells expressing SULF1 have diminished proliferation [75]. In their study, the expression of SULF1 was upregulated in the cells cultured in serum medium, suggesting a possibility that also in hESCs, SULF1 expression may diminish cell proliferation rate. They also found that CER1 was expressed only in the cells cultured in serum medium. CER1 inhibits Nodal signaling during embryonic development in mouse, and cell proliferation is inhibited in the same regions where CER1 is expressed [76], thus suggesting that CER1 might have similar effect in hESCs, i.e. reduced cell proliferation in serum medium. In addition, over 100 genes were found to be significantly differentially expressed between hESCs cultured in serum-containing medium and those cultured in KOSR medium. For example, this study revealed that a group of 50 adhesion-related genes (including integrins, laminin receptors, and TGFBR1) were upregulated in hESCs cultured under FCS-containing medium, consistent with the observed decrease in adhesion of hESCs grown in KOSR. Such changes may have fundamental importance for the selection process in vitro prolonged hESC culture period. In addition, we recently reported that the tumor necrosis superfamily member $\mathrm{CD} 30$, a surface biomarker for malignant cells in Hodgkin's disease and EC cells [77, 78], is induced through an epigenetic mechanism by ascorbate contained in KOSR medium [79]. We further showed that CD30 signaling enhances single cell survival and leads to transcriptome changes related to cell signalling, lipid metabolism and tissue development. Collectively, these reports provide evidence of altered $\mathrm{hESC}$ behavior as a result of culture induced changes in hESCs following prolonged culture in vitro.

\section{EPIGENETIC INSTABILITY OF HUMAN PLURIPOTENT STEM CELLS}

\section{Occurrence of Epigenetic Changes in Human Pluripotent Stem Cells in vitro}

Although genetic instability of hESCs has received some attention, little is know about their epigenome. It has been proposed by Baylin et al. that epigenetic changes precede genetic alterations and predispose cells to malignant transformation [70]. This implies that epigenetic changes may precede and contribute to the genetic instability during culture. Epigenetic changes may therefore have profound implications for the use of hESCs in regenerative medicine by affecting differentiation capacity and tumorgenic potential [7].

DNA methylation defects can affect different components of the genome, including $\mathrm{CpG}$ islands in promoter regions, repetitive sequences, and imprinted genes [80]. Methylation alteration in imprinted genes can be induced by cellular stress [81] and specific culture conditions [7]. A recent study by Rugg-Gunn et al. showed that one line of four examined showed evidence of imprinting instability in undifferentiated hESCs and only at high passage number (p66-p101) [82]. Another recent study by Pick et al. [83] shows that abnormal expression of imprinted genes associated with DNA demethylation occurs in different iPSC lines at various levels. In addition, a more extensive study by Rugg-Gunn et al. as part of the International Stem Cell Initiative, reported that some hESCs show loss of allelespecific expression, which could have implication for hESC differentiation and epigenetic stability [84]. For example, they found that the imprinting status of $H 19, I G F 2$ and $M E G 3$ are highly variable. The reasons for this variability among different cell lines remain unclear. Loss of stable imprinted gene expression, leading to bi-allelic expression, can change the dosage of the corresponding gene products and the relative biochemical activities of the pathways they mediate. Thus, depending on the gene in question, this increase in expression may result in changes in hESC behavior and/or differentiation. For example, a recent report showed that IGF2 may be an important survival factor for hESCs; therefore, hESCs with increased IGF2 expression (caused by upregulation of transcription and/or loss of imprint stability to biallelic expression) may result in a selective advantage in culture.

Alteration of DNA methylation at non-imprinted genome loci can induce genomic instability and is associated with cancer $[85,86]$. Both DNA hypo-methylation (activating oncogenes) and hyper-methylation (inactivating tumor suppressors) at diverse genomic sequences are found in tumors [87]. Indeed Allegrucci et al. showed the occurrence of epigenetic instability in hESCs by examining the DNA methylation profiles of $>2000$ genomic loci by Restriction Landmark Genome Scanning [88]. Six independently derived hESC lines are found to inherit further epigenetic changes over time in culture, with most changes arising in the earliest stages post-derivation. Notably, the loci affected varied between lines. The majority of culture-induced changes were losses of DNA methylation. The epigenetic changes $(82.3-87.5 \%)$ were stably inherited both within the 
undifferentiated cells and post-differentiation. They further reported that adapting a line to a serum-free culture system resulted in additional epigenetic instability. Overall $80.5 \%$ of the unstable loci uncovered in hESCs have been associated previously with an adult tumor phenotype. Their study strongly suggested that current methods of hESC propagation can rapidly program stable and unpredictable epigenetic changes in the stem cell genome.

Epigenetic variation between hESCs may also perturb $\mathrm{X}$ chromosome inactivation. $\mathrm{X}$ chromosome inactivation is required for dosage compensation of $\mathrm{X}$-linked genes in female cells [89]. A few studies have reported variable failure of $\mathrm{X}$ chromosome inactivation in female hESCs. For example, Hoffman et al. observed the XIST expression indicative of X -inactivation in both undifferentiated and differentiated cells of H9 and CyT25, but not H7 [90]. Sperger et al. reported that $\mathrm{H} 7$ and $\mathrm{H} 13$ cell lines also expressed XIST in the undifferentiated state, whereas H9 did not [91]. Another report by Dhara et al. showed the variation within a cell line; undifferentiated $\mathrm{H} 9$ cells in an independent study exhibited only active $\mathrm{X}$ chromosomes, although $\mathrm{X}$-inactivation was established after differentiation [92]. Furthermore, undifferentiated $\mathrm{H} 7$ cells demonstrated XIST expression that was lost in high passage aneuploid cells from the same line, even after their differentiation [35]. It is not clear why differences in $\mathrm{X}$ chromosome inactivation (XCI) between hESC lines are observed.

Kathrin Plath and colleagues [93] have characterized XCI status upon reprogramming of female human somatic cells into female hIPCs and have shown consistently that completely reprogrammed hiPSCs, derived under standard b-FGF culture conditions, carry an inactive $\mathrm{X}$-chromosome (Xi) with XIST RNA coating and classic markers of Xi heterochromatin. This mutually exclusive, nonrandom pattern of XCI among hiPSC lines is consistent with the notion that the $\mathrm{Xi}$ does not reactivate during reprogramming of human somatic cells and that a single cell from the mosaic fibroblast popu-

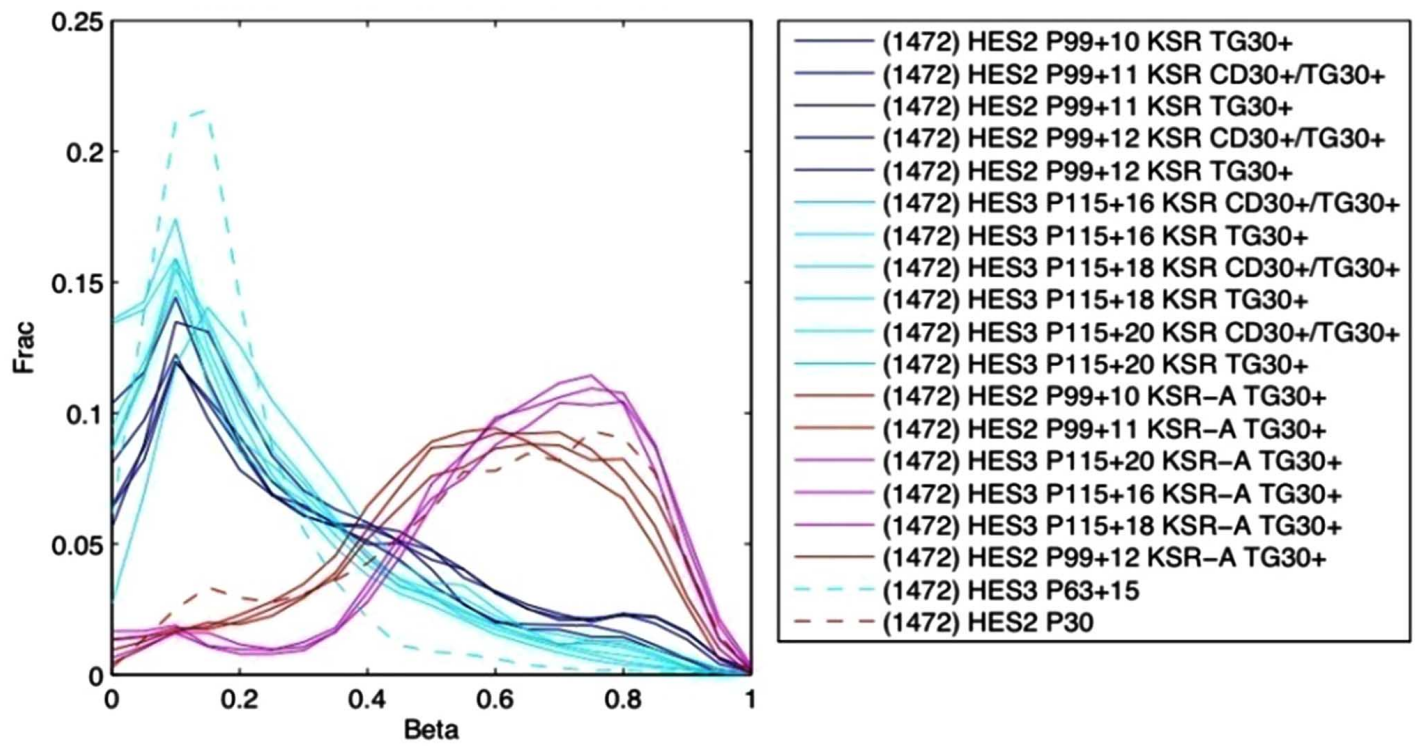

Fig. (1). Overall methylation levels are decreased in ascorbate-treated samples. Among 1,472 highly variable probes, the vast majority were methylated in ASC- samples (red, pink) and unmethylated in ASC+ (blue,cyan) samples. Notice that HES3 P63+15 (dotted cyan line), cells cultured in ASC + media for a large number of passages, have significantly less methylation than the rest of the ASC + lines which were cultured in ascorbate for between 10-20 passages. Abbreviations: KSR, knock-out serum replacement; KSR-A, knock-out serum replacement without ascorbate; TG30+, TG30+ FACS-sorted; CD30+/TG30+, CD30+ and TG30+ FACS-sorted. lation that has silenced either the maternally or the paternally inherited $\mathrm{X}$ chromosome gives rise to the reprogramming event. Most notably though, upon continued expansion in culture, female hiPSCs are prone to lose XIST expression, probably through methylation of its promoter, similar to what has been observed with female hESCs [94-97]. The absence of XIST RNA coating leads to the loss of XISTdependent chromatin marks, but it does not reactivate the $\mathrm{Xi}$ and the possibility of partial derepression of the $\mathrm{X}$ chromosome accompanying XIST loss must not be excluded. The impact of culture conditions on XCI has been clearly shown by Lengner and colleagues [94] who described the derivation of hESCs with two active $\mathrm{X}$ chromosomes (Xas) from blastocysts under physiological oxygen conditions and clearly demonstrate that hESCs with two Xas initiate XCI in response to different kinds of cellular stress, including exposure to atmospheric oxygen levels, possibly explaining why most hESC lines cultured in labs to date carry an $\mathrm{Xi}$. Thus, it appears likely that the epiblast progenitor cells of the human blastocyst have two Xas and that cellular stress during derivation and especially maintenance of hESCs in vitro leads to progressive perturbations of this epigenetic state and precocious XCI in the undifferentiated state.

Furthermore, during the process of iPSC generation, the culture of emerging iPSC clones under serum free culture conditions is thought to either induce further epigenetic modifications that will sustain undifferentiated growth of pluripotent cells or select for iPSCs that possess such epigenetic changes [98].

\section{Culture Induced Epigenetic Changes in vitro}

Our group, for the first time, demonstrated that ascorbate, a medium component in the widely used KOSR medium for hESC and iPSC culture, causes widespread, consistent and remarkably specific DNA demethylation of 1,847 genes in hESCs (Fig. 1), including important stem cell genes such as Jagged-2, Lefty2, Piwil2, Cdx4 and Fox [99]. Previously 
Allegrucci et al. [88] reported that switching BG01 hESC cultures from serum-containing (ascorbate-free) standard conditions to serum-free or feeder-free (ascorbate-containing) conditions resulted in epigenetic changes regardless of passage methods. These mainly comprised DNA methylation losses. Our data showing vitamin C induces DNA demethylation provides a rationale for this observation. In addition, specific DNA demethylation of the hESC epigenome by ascorbate significantly overlaps with bivalent domains [100] and gene sets that are demethylated during reprogramming
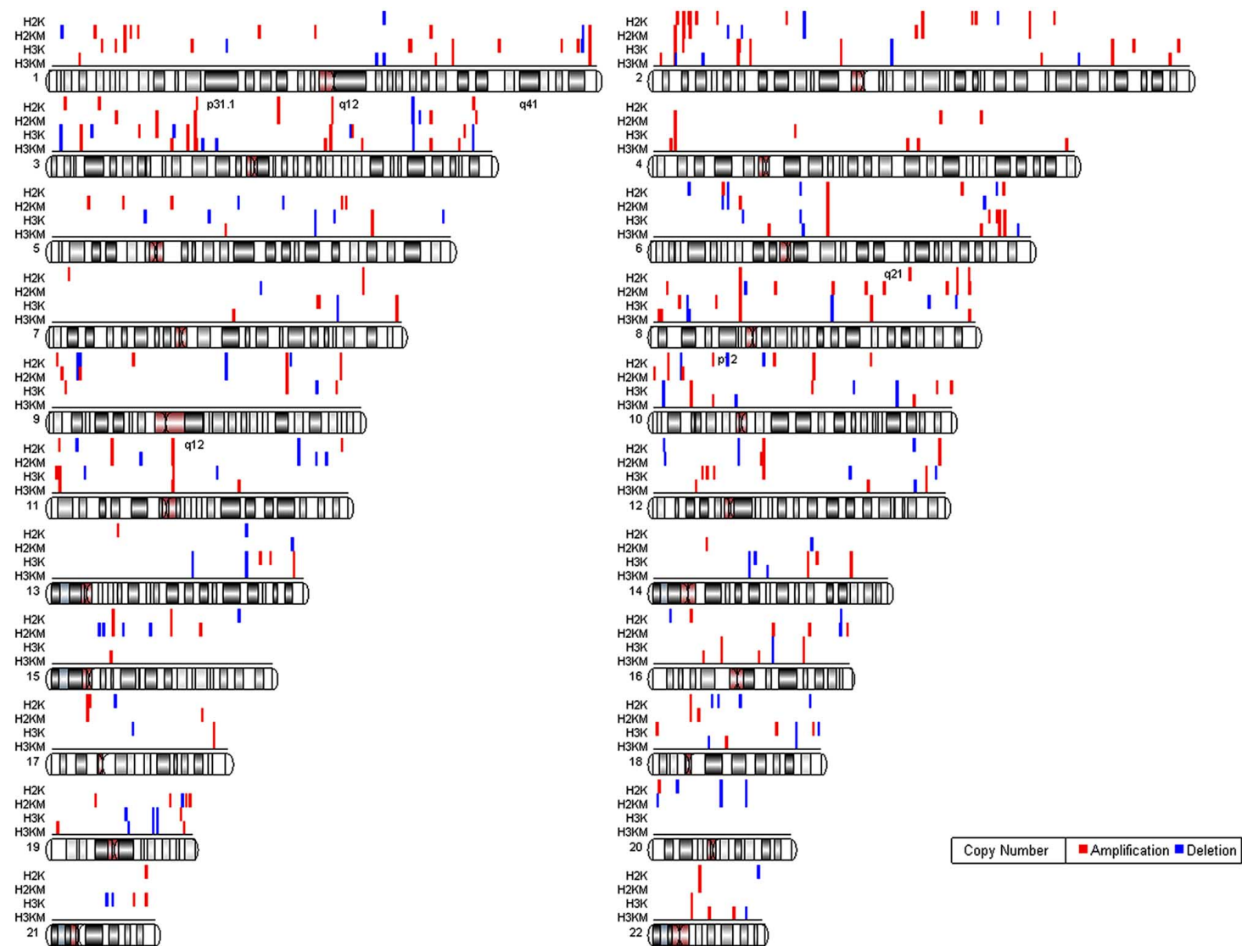

Fig. (2). Analysis of genome-wide copy number variation of hESCs cultured with ascorbate or without ascorbate using illumina 610-Quad beadchip. H2K, HES2 hESC line with ascorbate; H2KM, HES2 without ascorbate; H3K, HES3 with ascorbate; H3KM, HES3 without ascorbate; red, region with copy number amplification; blue, region with copy number deletion.

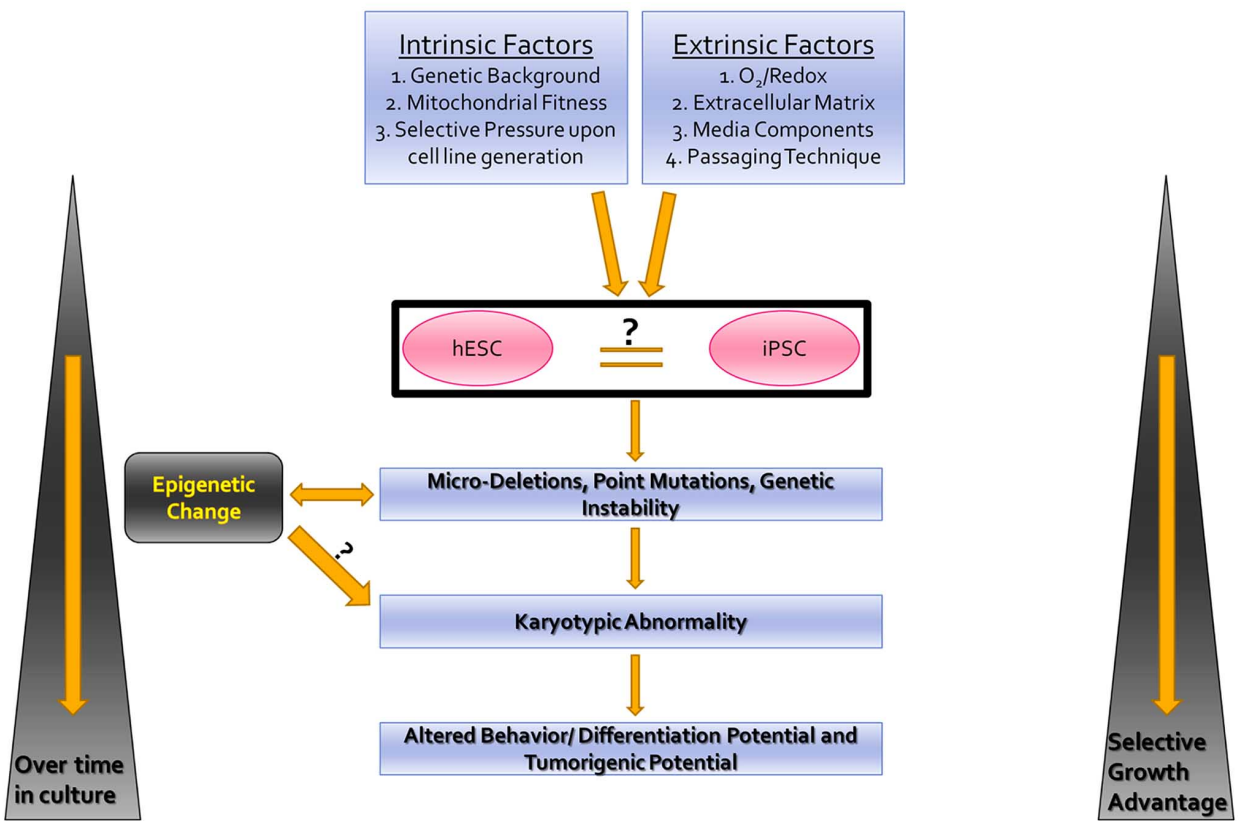

Fig. (3). A stochastic model of the intrinsic and especially extrinsic factors that are most likely causative of culture-induced genetic and epigenetic abnormalities in hESCs and hiPSCs over increasing period of time. 
[101]. Furthermore, we have not found hESCs cultured with ascorbate display more micro-deletion or amplification on the genome than those cultured without ascorbate (Fig. 2, unpublished data). Therefore, ascorbate-mediated DNA demethylation might affect differentiation capacity of pluripotent stem cells.

\section{CONCLUSION}

It has become clear now that both hESCs [88, 99] and iPSCs [83, 98] undergo progressive epigenetic changes during in vitro culture and that these changes can potentially affect both their genetic stability and their ability to efficiently differentiate into specific lineages (Fig. 3). Therefore, it seems increasingly evident that an extremely thorough analysis of genomic integrity must be carried out to identify even minor deletions, inversions or loss of individual alleles to absolutely ensure the safety of future human hESCs and iPSCs for regenerative medicine applications. Current characterization methods that involve simply traditional karyotype analysis are not sufficient. Taken together, all these observations highlight the need for (i) novel screening strategies to determine the experimental utility and biosafety of hESCs or iPSCs and (ii) optimization and standardization of procedures for the generation and culture of hESC and iPSC lines that minimize culture-induced epigenetic and genetic instability.

\section{REFERENCES}

[1] Thomson JA, Itskovitz-Eldor J, Shapiro SS, et al. Embryonic stem cell lines derived from human blastocysts. Science 1998; 282(5391): 1145-7.

[2] Draper JS, Smith K, Gokhale $\mathrm{P}$, et al. Recurrent gain of chromosomes $17 \mathrm{q}$ and 12 in cultured human embryonic stem cells. Nat Biotechnol 2004; 22(1): 53-4.

[3] Maitra A, Arking DE, Shivapurkar N, et al. Genomic alterations in cultured human embryonic stem cells. Nat Genet 2005; 37(10): 1099-103.

[4] Park IH, Arora N, Huo $\mathrm{H}$, et al. Disease-specific induced pluripotent stem cells. Cell 2008; 134(5): 877-86.

[5] Harrison NJ, Baker D, Andrews PW. Culture adaptation of embryonic stem cells echoes germ cell malignancy. Int J Androl 2007; 30(4): 275-81; discussion 281.

[6] Allegrucci C, Young LE. Differences between human embryonic stem cell lines. Hum Reprod 2007; 13(2): 103-20.

[7] Allegrucci C, Denning C, Priddle H, Young L. Stem-cell consequences of embryo epigenetic defects. Lancet 2004; 364(9429): 206-8.

[8] Rajagopalan H, Lengauer C. Aneuploidy and cancer. Nature 2004; 432(7015): 338-41.

[9] Storchova Z, Pellman D. From polyploidy to aneuploidy, genome instability and cancer. Nat Rev Mol Cell Biol 2004; 5(1): 45-54.

[10] Brimble SN, Zeng X, Weiler DA, et al. Karyotypic stability, genotyping, differentiation, feeder-free maintenance, and gene expression sampling in three human embryonic stem cell lines derived prior to August 9, 2001. Stem Cells Dev 2004; 13(6): 58597.

[11] Cowan CA, Klimanskaya I, McMahon J, et al. Derivation of embryonic stem-cell lines from human blastocysts. N Engl J Med 2004; 350(13): 1353-6.

[12] Inzunza J, Sahlen S, Holmberg K, et al. Comparative genomic hybridization and karyotyping of human embryonic stem cells reveals the occurrence of an isodicentric X chromosome after longterm cultivation. Mol Hum Reprod 2004; 10(6): 461-6.

[13] Hanson C, Caisander G. Human embryonic stem cells and chromosome stability. Apmis 2005; 113(11-12): 751-5.
[14] Mitalipova MM, Rao RR, Hoyer DM, et al. Preserving the genetic integrity of human embryonic stem cells. Nat Biotechnol 2005; 23(1): 19-20

[15] Liu X, Wu H, Loring J, et al. Trisomy eight in ES cells is a common potential problem in gene targeting and interferes with germ line transmission. Dev Dyn 1997; 209(1): 85-91.

[16] Rosler ES, Fisk GJ, Ares X, et al. Long-term culture of human embryonic stem cells in feeder-free conditions. Dev Dyn 2004; 229(2): 259-74.

[17] Heins N, Englund MC, Sjoblom C, et al. Derivation, characterization, and differentiation of human embryonic stem cells. Stem Cells 2004; 22(3): 367-76.

[18] Caisander G, Park H, Frej K, et al. Chromosomal integrity maintained in five human embryonic stem cell lines after prolonged in vitro culture. Chromosome Res 2006; 14(2): 131-7.

[19] Kim SJ, Lee JE, Park JH, et al. Efficient derivation of new human embryonic stem cell lines. Mol Cells 2005; 19(1): 46-53.

[20] Nishikawa S, Goldstein RA, Nierras CR. The promise of human induced pluripotent stem cells for research and therapy. Nat Rev Mol Cell Biol 2008; 9(9): 725-9.

[21] Aasen T, Raya A, Barrero MJ, et al. Efficient and rapid generation of induced pluripotent stem cells from human keratinocytes. Nat Biotechnol 2008; 26(11): 1276-84

[22] Gonzalez F, Barragan Monasterio M, et al. Generation of mouseinduced pluripotent stem cells by transient expression of a single nonviral polycistronic vector. Proc Natl Acad Sci USA 2009; 106(22): 8918-22.

[23] Okita K, Nakagawa M, Hyenjong H, Ichisaka T, Yamanaka S. Generation of mouse induced pluripotent stem cells without viral vectors. Science 2008; 322(5903): 949-53.

[24] Yu J, Hu K, Smuga-Otto K, et al. Human induced pluripotent stem cells free of vector and transgene sequences. Science 2009; 324(5928): 797-801.

[25] Woltjen K, Michael IP, Mohseni P, et al. piggyBac transposition reprograms fibroblasts to induced pluripotent stem cells. Nature 2009; 458(7239): 766-70.

[26] Huangfu D, Osafune K, Maehr R, et al. Induction of pluripotent stem cells from primary human fibroblasts with only Oct4 and Sox2. Nat Biotechnol 2008; 26(11): 1269-75.

[27] Hong H, Takahashi K, Ichisaka T, et al. Suppression of induced pluripotent stem cell generation by the p53-p21 pathway. Nature 2009; 460(7259): 1132-5.

[28] Kawamura T, Suzuki J, Wang YV, et al. Linking the p53 tumour suppressor pathway to somatic cell reprogramming. Nature 2009; 460(7259): 1140-44.

[29] Li H, Collado M, Villasante A, et al. The Ink4/Arf locus is a barrier for iPS cell reprogramming. Nature 2009; 460(7259): 1136-9.

[30] Utikal J, Polo JM, Stadtfeld M, et al. Immortalization eliminates a roadblock during cellular reprogramming into iPS cells. Nature 2009; 460(7259): 1145-8.

[31] Marion RM, Strati K, Li H, et al. A p53-mediated DNA damage response limits reprogramming to ensure iPS cell genomic integrity. Nature 2009; 460(7259): 1149-53.

[32] Silva J, Barrandon O, Nichols J, Kawaguchi J, Theunissen TW, Smith A. Promotion of reprogramming to ground state pluripotency by signal inhibition. PLoS Biol 2008; 6(10): e253.

[33] Marion RM, Strati K, Li H, et al. A p53-mediated DNA damage response limits reprogramming to ensure iPS cell genomic integrity. Nature 2009; 460(7259): 1149-53.

[34] Baker DE, Harrison NJ, Maltby E, et al. Adaptation to culture of human embryonic stem cells and oncogenesis in vivo. Nat Biotechnol 2007; 25(2): 207-15.

[35] Enver T, Soneji S, Joshi C, et al. Cellular differentiation hierarchies in normal and culture adapted human embryonic stem cells. Hum Mol Genet 2005; 14(21): 3129-40.

[36] Plaia TW, Josephson R, Liu Y, et al. Characterization of a new NIH-registered variant human embryonic stem cell line, BG01V: a tool for human embryonic stem cell research. Stem Cells 2006; 24(3): 531-46.

[37] Herszfeld D, Wolvetang E, Langton-Bunker E, et al. CD30 is a survival factor and a biomarker for transformed human pluripotent stem cells. Nat Biotechnol 2006; 24: 351-7.

[38] Xiao L, Yuan X, Sharkis SJ. Activin A maintains self-renewal and regulates fibroblast growth factor, Wnt, and bone morphogenic protein pathways in human embryonic stem cells. Stem Cells 2006; 24(6): 1476-86 
[39] Andrews PW, Matin MM, Bahrami AR, Damjanov I, Gokhale P, Draper JS. Embryonic stem (ES) cells and embryonal carcinoma (EC) cells: opposite sides of the same coin. Biochem Soc Trans 2005; 33(Pt 6): 1526-30.

[40] Oosterhuis JW, Looijenga LH. Current views on the pathogenesis of testicular germ cell tumours and perspectives for future research: highlights of the 5th Copenhagen Workshop on Carcinoma in situ and Cancer of the Testis. Apmis 2003; 111(1): 280-9.

[41] Damjanov I. Pathogenesis of testicular germ cell tumours. Eur Urol 1993; 23(1): 2-5; discussion 6-7.

[42] Looijenga LH, Oosterhuis JW. Pathogenesis of testicular germ cell tumours. Rev Reprod 1999; 4(2): 90-100.

[43] Turnpenny L, Brickwood S, Spalluto CM, et al. Derivation of human embryonic germ cells: an alternative source of pluripotent stem cells. Stem Cells 2003; 21(5): 598-609.

[44] Shamblott MJ, Axelman J, Wang S, et al. Derivation of pluripotent stem cells from cultured human primordial germ cells. Proc Natl Acad Sci USA 1998; 95(23): 13726-31.

[45] Park JH, Kim SJ, Lee JB, et al. Establishment of a human embryonic germ cell line and comparison with mouse and human embryonic stem cells. Mol Cells 2004; 17(2): 309-15.

[46] Tirkkonen M, Tanner M, Karhu R, Kallioniemi A, Isola J, Kallioniemi OP. Molecular cytogenetics of primary breast cancer by CGH. Genes Chromosomes Cancer 1998; 21(3): 177-84.

[47] Azuhata T, Scott D, Takamizawa S, et al. The inhibitor of apoptosis protein survivin is associated with high-risk behavior of neuroblastoma. J Pediatr Surg 2001; 36(12): 1785-91.

[48] Ishitani K, Yoshida T, Kitagawa H, Ohta H, Nozawa S, Kato S. p54nrb acts as a transcriptional coactivator for activation function 1 of the human androgen receptor. Biochem Biophys Res Commun 2003; 306(3): 660-5

[49] Bai VU, Cifuentes E, Menon M, Barrack ER, Reddy GP. Androgen receptor regulates $\mathrm{Cdc} 6$ in synchronized $\mathrm{LNCaP}$ cells progressing from G1 to S phase. J Cell Physiol 2005; 204(2): 381-7.

[50] Secombe J, Pierce SB, Eisenman RN. Myc: a weapon of mass destruction. Cell 2004; 117(2): 153-6.

[51] Rubio D, Garcia-Castro J, Martin MC, et al. Spontaneous human adult stem cell transformation. Cancer Res 2005; 65(8): 3035-9.

[52] Singh KK. Mitochondrial dysfunction is a common phenotype in aging and cancer. Ann NY Acad Sci 2004; 1019: 260-4.

[53] Czarnecka AM, Golik P, Bartnik E. Mitochondrial DNA mutations in human neoplasia. J Appl Genet 2006; 47(1): 67-78.

[54] Thomson A, Wojtacha D, Hewitt Z, et al. Human embryonic stem cells passaged using enzymatic methods retain a normal karyotype and express CD30. Cloning Stem Cells 2008; 10(1): 89-106.

[55] Werbowetski-Ogilvie TE, Bosse M, Stewart $\mathrm{M}$, et al. Characterization of human embryonic stem cells with features of neoplastic progression. Nat Biotechnol 2009; 27(1): 91-7.

[56] Pera MF. Unnatural selection of cultured human ES cells? Nat Biotechnol 2004; 22(1): 42-3.

[57] Speicher MR, Carter NP. The new cytogenetics: blurring the boundaries with molecular biology. Nat Rev Genet 2005; 6(10): 782-92.

[58] Matsui Y, Zsebo K, Hogan BL. Derivation of pluripotential embryonic stem cells from murine primordial germ cells in culture. Cell 1992; 70(5): 841-7.

[59] Rajpert-De Meyts E. Developmental model for the pathogenesis of testicular carcinoma in situ: genetic and environmental aspects. Hum Reprod Update 2006; 12(3): 303-23.

[60] Ekbom A. Growing evidence that several human cancers may originate in utero. Semin Cancer Biol 1998; 8(4): 237-44.

[61] Levenstein ME, Ludwig TE, Xu RH, et al. Basic fibroblast growth factor support of human embryonic stem cell self-renewal. Stem Cells 2006; 24(3): 568-74

[62] Hong Y, Stambrook PJ. Restoration of an absent G1 arrest and protection from apoptosis in embryonic stem cells after ionizing radiation. Proc Natl Acad Sci USA 2004; 101(40): 14443-8.

[63] Fluckiger AC, Marcy G, Marchand M, et al. Cell cycle features of primate embryonic stem cells. Stem Cells 2006; 24(3): 547-56.

[64] Damelin M, Sun YE, Sodja VB, Bestor TH. Decatenation checkpoint deficiency in stem and progenitor cells. Cancer Cell 2005; 8(6): 479-84.

[65] Mantel C, Guo Y, Lee MR, et al. Checkpoint-apoptosis uncoupling in human and mouse embryonic stem cells: a source of karyotpic instability. Blood 2007; 109(10): 4518-27.
[66] Buzzard JJ, Gough NM, Crook JM, Colman A. Karyotype of human ES cells during extended culture. Nat Biotechnol 2004; 22(4): 381-2; author reply 382

[67] Xu C, Inokuma MS, Denham J, et al. Feeder-free growth of undifferentiated human embryonic stem cells. Nat Biotechnol 2001; 19(10): 971-4.

[68] Wong RC, Pebay A, Nguyen LT, Koh KL, Pera MF. Presence of functional gap junctions in human embryonic stem cells. Stem Cells 2004; 22(6): 883-9.

[69] Zeng X, Miura T, Luo Y, et al. Properties of pluripotent human embryonic stem cells BG01 and BG02. Stem Cells 2004; 22(3): 292-312.

[70] Baylin SB, Ohm JE. Epigenetic gene silencing in cancer - a mechanism for early oncogenic pathway addiction? Nat Rev Cancer 2006; 6(2): 107-16.

[71] von Zglinicki T, Pilger R, Sitte N. Accumulation of single-strand breaks is the major cause of telomere shortening in human fibroblasts. Free Radic Biol Med 2000; 28(1): 64-74.

[72] Forsyth NR, Musio A, Vezzoni P, Simpson AH, Noble BS, McWhir J. Physiologic oxygen enhances human embryonic stem cell clonal recovery and reduces chromosomal abnormalities. Cloning Stem Cells 2006; 8(1): 16-23.

[73] Koivisto H, Hyvarinen M, Stromberg AM, et al. Cultures of human embryonic stem cells: serum replacement medium or serumcontaining media and the effect of basic fibroblast growth factor. Reprod Biomed Online 2004; 9(3): 330-7.

[74] Skottman H, Stromberg AM, Matilainen E, Inzunza J, Hovatta O, Lahesmaa R. Unique gene expression signature by human embryonic stem cells cultured under serum-free conditions correlates with their enhanced and prolonged growth in an undifferentiated stage. Stem Cells 2006; 24(1): 151-67.

[75] Lai J, Chien J, Staub J, et al. Loss of HSulf-1 up-regulates heparinbinding growth factor signaling in cancer. J Biol Chem 2003; 278(25): 23107-17.

[76] Yamamoto M, Saijoh Y, Perea-Gomez A, et al. Nodal antagonists regulate formation of the anteroposterior axis of the mouse embryo. Nature 2004; 428(6981): 387-92.

[77] Durkop H, Foss HD, Eitelbach F, et al. Expression of the CD30 antigen in non-lymphoid tissues and cells. J Pathol 2000; 190(5): 613-8.

[78] Pera MF, Bennett W, Cerretti DP. Expression of CD30 and CD30 ligand in cultured cell lines from human germ-cell tumors. Lab Invest 1997; 76(4): 497-504.

[79] Chung TL, Turner JP, Thaker N, et al. Ascorbate Promotes Epigenetic Activation of CD30 in Human Embryonic Stem Cells. Stem Cells 2010; 28(10): 1782-93.

[80] Robertson KD. DNA methylation and human disease. Nat Rev Genet 2005; 6(8): 597-610.

[81] Pantoja C, de Los Rios L, Matheu A, Antequera F, Serrano M. Inactivation of imprinted genes induced by cellular stress and tumorigenesis. Cancer Res 2005; 65(1): 26-33.

[82] Rugg-Gunn PJ, Ferguson-Smith AC, Pedersen RA. Epigenetic status of human embryonic stem cells. Nat Genet 2005; 37(6): 585 7 .

[83] Pick M, Stelzer Y, Bar-Nur O, Mayshar Y, Eden A, Benvenisty N. Clone- and gene-specific aberrations of parental imprinting in human induced pluripotent stem cells. Stem Cells 2009; 27(11): 2686-90.

[84] Adewumi O, Aflatoonian B, Ahrlund-Richter L, et al. Characterization of human embryonic stem cell lines by the International Stem Cell Initiative. Nat Biotechnol 2007; 25(7): 80316 .

[85] Gaudet F, Hodgson JG, Eden A, et al. Induction of tumors in mice by genomic hypomethylation. Science 2003; 300(5618): 489-92.

[86] Herman JG, Baylin SB. Gene silencing in cancer in association with promoter hypermethylation. N Engl J Med 2003; 349(21): 2042-54.

[87] Jones PA, Baylin SB. The fundamental role of epigenetic events in cancer. Nat Rev Genet 2002; 3(6): 415-28.

[88] Allegrucci C, Wu YZ, Thurston A, et al. Restriction landmark genome scanning identifies culture-induced DNA methylation instability in the human embryonic stem cell epigenome. Hum Mol Genet 2007; 16(10): 1253-68.

[89] Heard E, Disteche CM. Dosage compensation in mammals: finetuning the expression of the X chromosome. Genes Dev 2006 ; 20(14): 1848-67. 
[90] Hoffman LM, Carpenter MK. Characterization and culture of human embryonic stem cells. Nat Biotechnol 2005; 23(6): 699-708.

[91] Sperger JM, Chen X, Draper JS, et al. Gene expression patterns in human embryonic stem cells and human pluripotent germ cell tumors. Proc Natl Acad Sci USA 2003; 100(23): 13350-5.

[92] Dhara SK, Benvenisty N. Gene trap as a tool for genome annotation and analysis of $\mathrm{X}$ chromosome inactivation in human embryonic stem cells. Nucleic Acids Res 2004; 32(13): 3995-4002.

[93] Tchieu J, Kuoy E, Chin MH, et al. Female Human iPSCs Retain an Inactive X Chromosome. Cell Stem Cell 2010; 7(3): 329-42.

[94] Lengner CJ, Gimelbrant AA, Erwin JA, et al. Derivation of pre-X inactivation human embryonic stem cells under physiological oxygen concentrations. Cell 2010; 141(5): 872-83.

[95] Hall LL, Byron M, Butler J, et al. X-inactivation reveals epigenetic anomalies in most hESC but identifies sublines that initiate as expected. J Cell Physiol 2008; 216(2): 445-52.

[96] Shen Y, Matsuno Y, Fouse SD, et al. X-inactivation in female human embryonic stem cells is in a nonrandom pattern and prone to epigenetic alterations. Proc Natl Acad Sci USA 2008; 105(12): 4709-14.

[97] Silva J, Mak W, Zvetkova I, et al. Establishment of histone h3 methylation on the inactive $\mathrm{X}$ chromosome requires transient recruitment of Eed-Enx1 polycomb group complexes. Dev Cell 2003; 4(4): 481-95.

[98] Chin MH, Mason MJ, Xie W, et al. Induced pluripotent stem cells and embryonic stem cells are distinguished by gene expression signatures. Cell Stem Cell 2009; 5(1): 111-23.

[99] Chung TL, Brena RM, Kolle G, et al. Vitamin C Promotes Widespread Yet Specific DNA Demethylation of the Epigenome in Human Embryonic Stem Cells. Stem Cells 2010; 28(10): 1848-55.

[100] Guenther MG, Levine SS, Boyer LA, Jaenisch R, Young RA. A chromatin landmark and transcription initiation at most promoters in human cells. Cell 2007; 130(1): 77-88.

[101] Doi A, Park IH, Wen B, et al. Differential methylation of tissueand cancer-specific $\mathrm{CpG}$ island shores distinguishes human induced pluripotent stem cells, embryonic stem cells and fibroblasts. Nat Genet 2009; 41(12): 1350-53.

Received: August 26, 2010

Revised: August 28, 2010

Accepted: August 28, 2010

(c) Chung et al.; Licensee Bentham Open.

This is an open access article licensed under the terms of the Creative Commons Attribution Non-Commercial License (http://creativecommons.org/licenses/by$\mathrm{nc} / 3.0 /$ ), which permits unrestricted, non-commercial use, distribution and reproduction in any medium, provided the work is properly cited. 